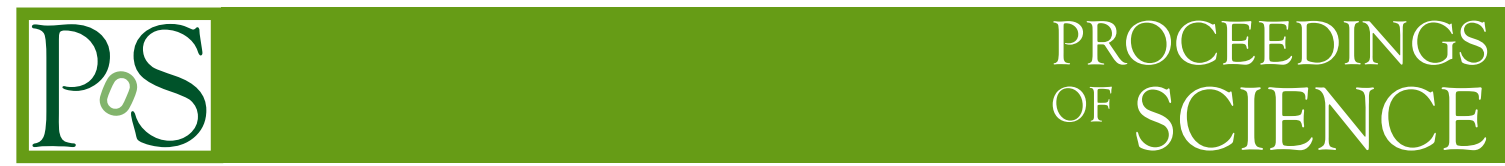

\title{
Infrared confinement and meson spectroscopy
}

\section{Gurjav Ganbold*}

Bogoliubov Laboratory of Theoretical Physics, JINR, Dubna;

Institute of Physics and Technology, MAS, Ulaanbaatar

E-mail: ganboldetheor.jinr.ru

Quark-antiquark bound states are studied within a relativistic quantum-field model based on the infrared confinement. The conventional meson spectrum is determined by a master equation similar to the ladder Bethe-Salpeter equation. Masses of light, intermediate and heavy mesons are estimated in a wide range of scale (up to $10 \mathrm{GeV}$ ). In doing so we revealed a new, specific infrared-finite behavior of the QCD effective coupling in the low-energy domain.

XXI International Baldin Seminar on High Energy Physics Problems September 10-15, 2012

JINR, Dubna, Russia

${ }^{*}$ Speaker. 


\section{Introduction}

Nowadays, many interesting and novel behavior in particle physics is expected in the infrared (IR) region at low energies below $Q \sim 1 \mathrm{GeV}$. Therefore, the study of QCD behavior at large distances remains an active field of research. A correct description of hadron dynamics in the IR domain is required to understand a number of phenomena such as quark confinement, hadronization of quark-gluon matter and the QCD running coupling, etc. Hereby, the conventional perturbation theory cannot be effectively used in the IR region (see, e.g., [四, [ [, []]).

One of the fundamental parameters of nature, the QCD effective coupling $\alpha_{s}$ and its behavior in the IR region are the subject of intensive studies in both theoretical and experimental particle physics. As is known, QCD predicts a dependence of the physical coupling $g$ under changes of distance $\sim 1 / Q$. This dependence $\alpha_{s}(Q) \doteq g^{2} /(4 \pi)$ is described theoretically by the renormalization group equations and determined experimentally at relatively high energies [ [G, [0]. Recent developments on this way were summarized in a number of articles (e.g., [6, —, 8] ). There exists a phenomenological indication in favor of a smooth transition from short distance to long distance physics [四].

Many quantities in particle physics are affected by the IR behavior of the coupling in different amounts. Nevertheless, the long-distance behavior of $\alpha_{s}$ is not well defined, it needs to be more specified [ए, [1, [2] and correct description of QCD effective coupling in the IR regime remains one of the actual problems in particle physics.

\section{Model}

The dependence of $\alpha_{s}$ on mass scale $M$ in the low-energy region may be determined by exploiting the hadron spectrum $[\mathbb{[ 1 3}]$. In doing so, we define the meson masses by solving the master equation (which is similar to the ladder Bethe-Salpeter equation) for two-quark bound states within a relativistic field model [14]].

For the spectra of two-quark bound states we develop a relativistic quantum-field model based on IR confinement and consider the model Lagrangian:

$$
\mathscr{L}=-\frac{1}{4}\left(F_{\mu v}^{A}-g f^{A B C} \mathscr{A}_{\mu}^{B} \mathscr{A}_{v}^{C}\right)^{2}+\left(\bar{q}_{f}^{a}\left[\gamma_{\alpha} \partial^{\alpha}-m_{f}\right]^{a b} q_{f}^{b}\right)+g\left(\bar{q}_{f}^{a}\left[\Gamma_{C}^{\alpha} \mathscr{A}_{\alpha}^{C}\right]^{a b} q_{f}^{b}\right),
$$

where $\mathscr{A}_{\alpha}^{C}$ is the gluon field, $q_{f}^{a}$ - a quark field of flavor $f, F_{\mu \nu}^{A}=\partial^{\mu} \mathscr{A}_{v}^{A}-\partial^{v} \mathscr{A}_{\mu}^{A}$ and $\Gamma_{C}^{\alpha}=i \gamma_{\alpha} t^{C}$.

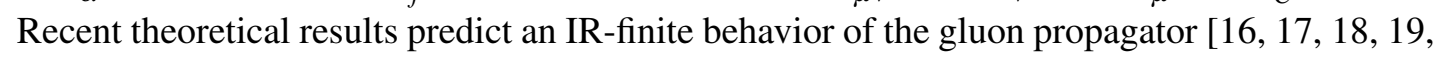
[20]. We consider a IR-confined gluon propagator in Feynman gauge as follows [प15]:

$$
\tilde{D}_{\mu v}^{A B}(p)=\delta^{A B} \delta_{\mu v} \int_{0}^{1 / \Lambda^{2}} d s e^{-s p^{2}}=\delta^{A B} \delta_{\mu v} \frac{1-\exp \left(-p^{2} / \Lambda^{2}\right)}{p^{2}} .
$$

Here, an IR parametrization is hidden in the confinement scale $\Lambda$. The gluon propagator exhibits an explicit IR-finite behavior $\tilde{D}(0) \sim 1 / \Lambda^{2}$. Deconfinement appears as $\Lambda \rightarrow 0$.

Similarly, the IR-confined quark propagator reads:

$$
\tilde{S}_{m}^{a b}(\hat{p})=\delta^{a b} \frac{i \hat{p}+m_{f}}{\Lambda^{2}} \int_{0}^{1 / \Lambda^{2}} d t e^{-t\left(p^{2}+m_{f}^{2}\right)}=\delta^{a b} \frac{i \hat{p}+m_{f}}{p^{2}+m_{f}^{2}}\left\{1-\exp \left(-\frac{p^{2}+m_{f}^{2}}{\Lambda^{2}}\right)\right\} .
$$


Within the model the quark and gluon propagators $\tilde{S}(\hat{p})$ and $\tilde{D}(p)$ are entire analytic functions in the Euclidean space. The model parameters are the IR confinement scale $\Lambda$ and the constituent quark masses $m_{f}=\left\{m_{u d}, m_{s}, m_{c}, m_{b}\right\}$.

The leading-order contribution to the $(q \bar{q})$ bound states is determined by partition function [114]:

$$
\begin{aligned}
& Z_{q \bar{q}}=\iint \mathscr{D} \bar{q} \mathscr{D} q \exp \left\{-\left(\bar{q} S^{-1} q\right)+\frac{g^{2}}{2}\langle(\bar{q} \Gamma \mathscr{A} q)(\bar{q} \Gamma \mathscr{A} q)\rangle_{D}\right\} \\
& \langle(\bullet)\rangle_{D} \doteq \int \mathscr{D} \mathscr{A} e^{-\frac{1}{2}\left(\mathscr{A} D^{-1} \mathscr{A}\right)}(\bullet)
\end{aligned}
$$

1. First, we allocate the one-gluon exchange between colored biquark currents and isolate the color-singlet combinations.

$$
L_{2}=\frac{g^{2}}{2} \sum_{f_{1} f_{2}} \iint d x_{1} d x_{2}\left(\bar{q}_{f_{1}}\left(x_{1}\right) i \gamma_{\mu} t^{A} q_{f_{1}}\left(x_{1}\right)\right) D_{\mu v}^{A B}\left(x_{1}, x_{2}\right)\left(\bar{q}_{f_{2}}\left(x_{2}\right) i \gamma_{v} t^{B} q_{f_{2}}\left(x_{2}\right)\right) .
$$

2. Then, perform a Fierz transformation

$\left(i \gamma_{\mu}\right) \delta^{\mu v}\left(i \gamma_{v}\right)=\sum_{J} C_{J} \cdot O_{J} O_{J}, \quad C_{J}=\left\{1,1, \frac{1}{2},-\frac{1}{2}, 0\right\}, \quad O_{J}=\left\{I, i \gamma_{5}, i \gamma_{\mu}, \gamma_{5} \gamma_{\mu}, i \frac{\left[\gamma_{\mu}, \gamma_{v}\right]}{2}\right\}$

to extract different currents with $J=\{S, P, V, A, T\}$.

3. By introducing a system of orthonormalized functions $\left\{U_{Q}(x)\right\}$, where $Q=\left\{n_{r}, l, \mu\right\}$ are quantum numbers, we diagonalize the one-gluon exchange term on this system.

4. Then, we involve a Gaussian path-integral representation for the exponentials by introducing new auxiliary meson fields $B_{\mathscr{N}}$ with $\mathscr{N}=\left\{Q, J, f_{1}, f_{2}\right\}$. This allows us to take explicit path integration over quark variables.

5. We introduce a Hadronization Ansatz and do identify $B_{\mathscr{N}}(x)$ with meson fields carrying quantum numbers $\mathscr{N}$.

6. All quadratic field configurations $\left(\sim B_{\mathscr{N}}^{2}\right)$ in the 'kinetic' term should be isolated and we rewrite the partition function totally in terms of meson field variables as follows [पய4]:

$$
Z_{q \bar{q}} \rightarrow Z=\int \prod_{\mathscr{N}} \mathscr{D}_{\mathscr{N}} \exp \left\{-\frac{1}{2} \sum_{\mathscr{N} \mathscr{N}^{\prime}}\left(B_{\mathscr{N}}\left[\delta^{\mathscr{N}^{\prime}}+\alpha_{s} \lambda_{\mathscr{N} \mathscr{N}^{\prime}}\right] B_{\mathscr{N}^{\prime}}\right)-W_{\text {res }}\left[B_{\mathscr{N}}\right]\right\}
$$

where the residual part $W_{\text {res }}\left[B_{\mathscr{N}}\right] \sim 0\left(B_{\mathscr{N}}^{3}\right)$ describes interaction between mesons.

7. The Fourier transform of the leading-order term of the polarization operator reads

$\lambda_{J J^{\prime}}(p, x, y)=\frac{16 \pi}{9} \sqrt{C_{J} C_{J^{\prime}} D(x) D(y)} \int \frac{d^{4} k}{(2 \pi)^{4}} e^{-i k(x-y)} \operatorname{Tr}\left[O_{J} \tilde{S}_{m_{1}}\left(\hat{k}+\xi_{1} \hat{p}\right) O_{J^{\prime}} \tilde{S}_{m_{2}}\left(\hat{k}-\xi_{2} \hat{p}\right)\right]$,

where traces are taken on color and spinor indices. 
8. We diagonalize the polarization kernel on the orthonormal basis $\left\{U_{\mathscr{N}}\right\}$

$$
\iint d x d y U_{\mathscr{N}}(x) \lambda_{J J^{\prime}}(p, x, y) U_{\mathscr{N}^{\prime}}(y)=\delta^{\mathscr{N} \mathscr{N}^{\prime}} \lambda_{\mathscr{N}}\left(-p^{2}\right)
$$

that is equivalent to the solution of the corresponding ladder BSE.

9. In relativistic quantum-field theory a stable bound state of $n$ massive particles shows up as a pole in the $S$ matrix with a center of mass energy. Accordingly, the meson masses may be derived from the equation [2]]:

$$
1+\alpha_{S} \cdot \lambda_{\mathscr{N}}\left(M_{\mathscr{N}}^{2}\right)=0, \quad-p^{2}=M_{\mathscr{N}}^{2} .
$$

\section{Meson Spectrum}

The dependence of meson masses on $\alpha_{s}$ and model parameters is defined by Eq. ([2.7). Note, the kernel function $\lambda_{\mathscr{N}}$ is a real and finite, it allows us to derive both analytic and numeric solutions.

\subsection{Analytic Results}

By deriving Eq. (2.7), we reveal an asymptotical Regge-type behavior of meson squared masses:

$$
M_{J}^{2} \approx M_{0}^{2}+J \cdot \text { const }, \quad \text { for } \quad J>3 .
$$

Also, one can easily find that for the same quark content a vector meson is heavier than its pseudoscalar counterpart: $M_{V}>M_{P}$ because $C_{V}<C_{P}$.

\subsection{Numerical Estimates}

The polarization kernel $\lambda_{\mathscr{N}}$ is natively obtained real and symmetric that allows us to find a simple variational solution to this problem.

Further we exploit Eq. (2.]) in different ways, by solving either for $\alpha_{s}$ at given masses, or for $M_{J}$ at known values of $\alpha_{s}$. We adjust the model parameters by fitting experimental data for different values of confinement scale.

Then, we derive meson mass formula and adjust the model parameters by fitting heavy meson masses $(M \geq 2 \mathrm{GeV})$. Hereby, we obtain $\alpha_{s}$ from newest experimental data appeared in PDG2010 edition.

As a particular case, we choose $\Lambda=220 \mathrm{MeV}$ and fix a set of model parameters as follows:

$$
\begin{array}{r}
\Lambda=220 \mathrm{MeV}, \quad m_{u d}=247.2 \mathrm{MeV}, \\
m_{s}=432.5 \mathrm{MeV}, \quad m_{c}=1544.5 \mathrm{MeV}, \quad m_{b}=4740.9 \mathrm{MeV} .
\end{array}
$$

As an application, we also calculate some intermediate and heavy meson masses $(1<M<9.5$ $\mathrm{GeV}$ ). Our estimates of meson masses are shown in Table 1 . The relative error of our estimate does not exceed 2.5 percent in a wide range of mass. 


\begin{tabular}{|c|c||c|c||}
\hline$J^{P C}=0^{-+}$ & $M_{\mathbf{P}}$ & $J^{P C}=1^{--}$ & $M_{\mathbf{V}}$ \\
\hline$D(1870)$ & 1892 & $\rho(770)$ & 771 \\
$D_{s}(1970)$ & 1998 & $K^{*}(892)$ & 893 \\
$\eta_{c}(2980)$ & 3042 & $D^{*}(2010)$ & 1961 \\
$B(5279)$ & 5117 & $D_{s}^{*}(2112)$ & 2079 \\
$B_{s}(5370)$ & 5232 & $J / \Psi(3097)$ & 3097 \\
$B_{c}(6286)$ & 6238 & $B^{*}(5325)$ & 5168 \\
$\eta_{b}(9389)$ & 9384 & $\Upsilon(9460)$ & 9461 \\
\hline
\end{tabular}

Table 1. Estimated masses $M$ of conventional mesons (in $\mathrm{MeV}$ ) at confinement scale $\Lambda=220 \mathrm{MeV}$.

\section{QCD Running Coupling}

We consider the meson mass $M$ as an appropriate energy-scale parameter for coupling $\alpha_{s}(M)$.

Having adjusted model parameters, we estimate $\alpha_{s}(M)$ in the low-energy domain by exploiting meson masses below $\sim 1 \mathrm{GeV}$ [[13]. Then, we perform global evaluation of $\alpha_{s}(M)$ at the mass scale of conventional mesons (shown in Table 1) by using formula

$$
\alpha_{s}\left(M_{J}\right)=-1 / \lambda_{J}\left(M_{J}, \Lambda, m_{1}, m_{2}\right)
$$

and plot the resulting curve at $\Lambda=220 \mathrm{MeV}$ in Fig. 1 in comparison with recent low- and highenergy data of $\alpha_{s}(Q)$ [22].
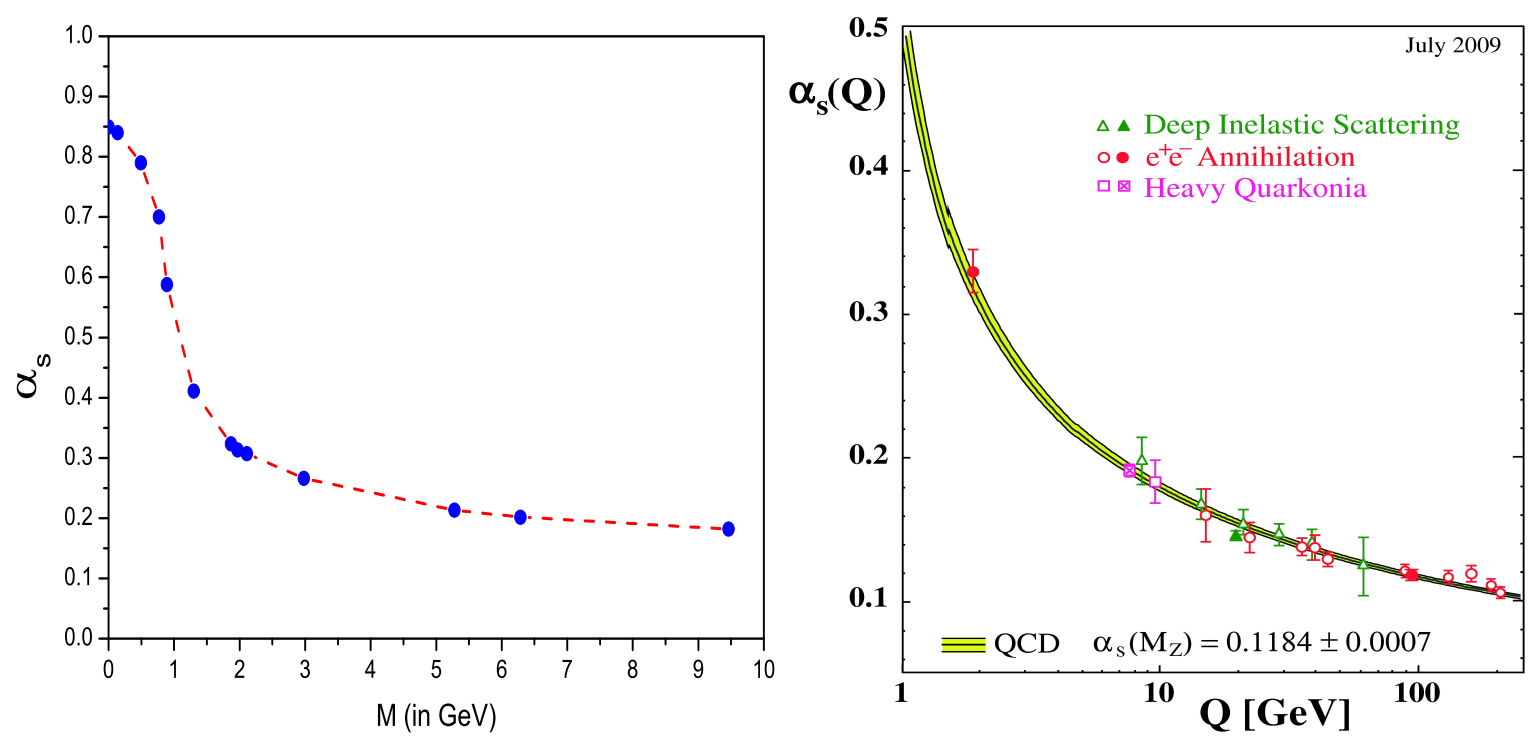

Fig. 1. Our estimate of $\alpha_{s}(M)$ at the confinement scale value $\Lambda=220 \mathrm{MeV}$ (left panel) compared with $\alpha_{s}(Q)$ defined in low- and high-energy experiments (for details see in [6, [22]). 


\section{IR-fixed Point of the QCD Running Coupling}

The possibility that the QCD coupling constant features an IR-finite behavior has been extensively studied in recent years (e.g., [22], 224, 225, 2] ]]).

By deriving Eq. ([2.]) for $M=0$ and $m_{1}=m_{2}=0$ we reveal a IR-fixed point

$$
\alpha_{s}^{0}=\alpha_{s}(0)=\frac{3 \pi}{16 \ln (2)} \approx 0.8498 \quad \Rightarrow \quad \alpha_{s}^{0} / \pi=0.2705
$$

We conclude that our result in Eq.(لل (ل) is in a reasonable agreement with often quoted estimates

$$
\begin{cases}\left\langle\alpha_{s}^{0} / \pi\right\rangle_{1 \mathrm{GeV}} \simeq 0.2 & {[\mathrm{[Q]},} \\ \alpha_{s}^{0} / \pi \simeq 0.19-0.25 & {[\mathrm{[R}],} \\ \alpha_{s}^{0} / \pi \simeq 0.265 & {[\mathrm{[Z7}],} \\ \alpha_{s}^{0} / \pi \simeq 0.26 & {[\mathrm{[Z]}]}\end{cases}
$$

and phenomenological evidences [22, 22]. The obtained IR-fixed value of the coupling constant is moderate.

To conclude, we demonstrate that global properties of the low-energy phenomena such as QCD running coupling and conventional meson spectrum may be explained reasonably in the framework of a simple relativistic quantum-field model of quark-gluon interaction based on infrared confinement.

We have demonstrated that the behavior of the QCD running coupling in the low-energy region (below $1 \mathrm{GeV}$ ) may be explained reasonably by using the meson data. Despite its model origin, the approximations used, and questions about the very definition of the coupling in the IR region, our approach exhibits a new, independent, and specific IR-finite behavior of QCD coupling. Besides, the model is able to address simultaneously different sections of the low-energy particle physics. Consideration can be extended to other problems such as exotic mesons, mixed and multiquark states, glueballs, baryons and hadronic decay processes.

\section{References}

[1] M. Baldicchi and G.M. Prosperi, [hep-ph/0310213]

[2] C.T.H. Davies et al., HPQCD Collab., Phys.Rev. D78 (2008) 114507.

[3] G. Corcella et al., JHEP 0101 (2001) 010.

[4] S. Chekanov et. al., Phys.Lett. B560 (2003) 7.

[5] S. Bethke, J. Phys. G26 (2000) R27 [hep-ex/0004021].

[6] Review of Particle Physics, K. Nakamura et al., J.Phys. G37 (2010) 075021.

[7] S. Bethke, Eur. Phys. J. C64 (2009) 689 [hep-ph/0908.1135].

[8] S. Bethke, Prog. Part. Nucl. Phys. 58 (2007) 351.

[9] Yu. L. Dokshitzer, G. Marchesini and B. R. Webber, Nucl. Phys. B469 (1996) 93.

[10] D.V. Shirkov, Theor. Math. Phys. 132 (2002) 1309. 
[11] A.V. Nesterenko, Int. J. M. Phys. A18 (2003) 5475.

[12] O. Kaczmarek and F. Zantow, Phys. Rev. D71 (2005) 114510.

[13] G. Ganbold, Phys. Rev. D81 (2010) 094008.

[14] G. Ganbold, Phys. Rev. D79 (2009) 034034.

[15] G. Ganbold, J.Phys.: Conf. Ser. 295 (2011) 012041.

[16] C. S. Fischer, R. Alkofer and H. Reinhardt, Phys. Rev. D65 (2002) 094008.

[17] C. Lerche and L. von Smekal, Phys. Rev. $D 65$ (2002) 125006.

[18] B. Alles et al., Nucl. Phys. B502 (1997) 325.

[19] H. Gies, Phys.Rev. D66 (2002) 025006.

[20] K. Langfeld, H. Reinhardt and J. Gattnar, Nucl. Phys. B621 (2002) 131.

[21] G. Ganbold, Phys. Part. Nucl. 43 (2012) 79.

[22] M. Baldicchi et al., Phys. Rev. D77 (2008) 034013.

[23] S. J. Brodsky and G. F. de Teramond, Phys. Lett. B582 (2004) 211.

[24] A. C. Aguilar, A. Mihara and A. A. Natale, Int. J. Mod. Phys. A19 (2004) 249.

[25] D. V. Shirkov and I. L. Solovtsov, Phys. Rev. Lett. 79 (1997) 1209;

D. V. Shirkov, Theor. Math. Phys. 136 (2003) 893 [hep-th/0210013].

[26] S. Godfrey and N. Isgur, Phys. Rev. D32 (1985) 189;

T. Barnes, F. E. Close and S. Monaghan, Nucl. Phys. B198 (1983) 380.

[27] T. Zhang and R. Koniuk, Phys. Lett. B261 (1991) 311;

C.R. Ji, F. Amiri, Phys. Rev. D42 (1990) 3764.

[28] F. Halzen, G. I. Krein and A. A. Natale, Phys. Rev. D47 (1993) 295.

[29] M. Baldicchi and G. M. Prosperi, Phys. Rev. D66 (2002) 074008. 